\title{
The Singularity of Orbital Measures on Compact Lie Groups
}

\author{
Kathryn E. Hare and Wai Ling Yee
}

\begin{abstract}
We find the minimal real number $k$ such that the $k$ th power of the Fourier transform of any continuous, orbital measure on a classical, compact Lie group belongs to $l^{2}$. This results from an investigation of the pointwise behaviour of characters on these groups. An application is given to the study of $L^{p}$-improving measures.
\end{abstract}

\section{Introduction}

It is well known that there are many continuous, singular measures on the circle (or any compact abelian group) all of whose convolution powers remain singular to $L^{1}$. In contrast, Ragozin in [5] proved the striking fact that if $G$ was a compact, connected, simple Lie group and $\mu$ was any central, continuous measure on $G$ then

$$
\mu^{\operatorname{dim} G} \in L^{1}(G)
$$

This fact obviously implies that the Fourier transform of any such measure tends to zero, and was used to prove that compact, simple Lie groups admit no infinite, central Sidon sets, again in contrast to the abelian case.

Ragozin's result was first improved in [2] where it was shown that if $k>\operatorname{dim} G / 2$ and $\mu$ was a continuous orbital measure on $G$ then $\mu^{k} \in L^{2}(G)$, while if $\mu$ was any central, continuous measure then $\mu^{k} \in L^{1}(G)$. In [3] estimates were made on the size of characters (in terms of their degrees) from which one could determine the minimal integer $k$ such that $\mu^{k} \in L^{2}$ for all continuous orbital measures $\mu$ on the classical, compact Lie groups.

2000 Mathematics Subject Classification: Primary 43A80; Secondary 22E46, 43A65. Keywords: orbital measures, compact Lie group, characters. 
Our interest in this paper is to determine the precise size of the Fourier transform of continuous orbital measures, i.e., to determine the minimal fractional power $k$ for which $\widehat{\mu}^{k} \in l^{2}$. The answer depends on the Lie group type and is summarized below.

Main Theorem Let $G$ be a compact, connected, simple Lie group of type $A_{n}, B_{n}, C_{n}$ or $D_{n}$. Then $\widehat{\mu}^{k} \in l^{2}$ for all continuous orbital measures $\mu$ on $G$ if and only if

$$
k>k_{0} \equiv \begin{cases}n+1 / 2 & \text { if } G \text { is type } A_{n} \\ 2 n-1 / 2 & \text { if } G \text { is type } B_{n} \\ n-1 / 4 & \text { if } G \text { is type } C_{n}, n \neq 3 \\ 13 / 4 & \text { if } G \text { is type } C_{3} \\ n-3 / 4 & \text { if } G \text { is type } D_{n} .\end{cases}
$$

The necessity of this choice of $k_{0}$ follows easily from the earlier work as is shown in Corollary 3.2.

The proof of sufficiency is divided into two parts. In the first part of the proof we continue the study of the pointwise behaviour of characters begun in [3]. This is relevant because if $\mu_{g}$ is the orbital measure associated to $g \in G$, then the Fourier transform of $\mu_{g}$ at the representation $\lambda$ is given by

$$
\widehat{\mu_{g}}(\lambda)=\operatorname{Tr} \lambda(g) / \operatorname{deg} \lambda .
$$

Here we show that for many $g \in G$ it is possible to improve the pointwise bounds of characters found in [3]. Using these improved estimates, the orbital measures corresponding to these points are easily seen to have the desired property. The arguments used to establish these statements are similar to those of [3]; the details are sketched in Section 3 and the results are summarized in Corollary 3.4. In the second part of the proof of sufficiency we consider the points $g \in G$ where the pointwise bounds found in [3] are sharp. For the orbital measures corresponding to these points a different, more direct approach to summing the Fourier transform is taken. This approach depends on properties of the particular points $g$ and is the content of Section 4.

Ricci and Stein in [6] proved that surface measures on compact, connected analytic manifolds which generate the group act as convolution operators from $L^{p}$ to $L^{2}$ for some $p<2$. In Section 5 we use our results to investigate the size of $p$ for the particular case of continuous orbital measures. Previously the minimal $p$ was determined for regular orbital measures in [7]. 


\section{Notation and Structural properties of Subroot Systems}

The proof of the theorem relies significantly on the representation theory of simple Lie groups. The main facts we need are recorded below.

\subsection{Notation and Basic Facts}

Let $G$ be a compact, connected, simple, non-exceptional Lie group of rank $n$. Let $Z(G)$ denote its centre and $W$ be its Weyl group. Denote by $e_{1}, \ldots, e_{m}$ the usual unit vectors in $R^{m}$ where $m=n+1$ in type $A_{n}$ and $m=n$ otherwise. We take a maximal torus $T$ with $\Phi$ the set of roots for $(G, T)$ described below.

$$
\begin{aligned}
& \text { Type Root system } \mathbf{\Phi} \\
& A_{n} \quad\left\{e_{i}-e_{j}: 1 \leq i \neq j \leq n+1\right\} \\
& B_{n} \quad\left\{ \pm e_{i}, \pm\left(e_{i} \pm e_{j}\right): 1 \leq i \neq j \leq n\right\} \\
& \text { Base } \boldsymbol{\Delta}=\left\{\alpha_{j}: j=1, \ldots, n\right\} \\
& \alpha_{j}=e_{j}-e_{j+1} \\
& \alpha_{j}=e_{j}-e_{j+1} \text { for } j \neq n \\
& \alpha_{n}=e_{n} \\
& C_{n} \quad\left\{ \pm 2 e_{i}, \pm\left(e_{i} \pm e_{j}\right): 1 \leq i \neq j \leq n\right\} \\
& \alpha_{j}=e_{j}-e_{j+1} \text { for } j \neq n \\
& \alpha_{n}=2 e_{n} \\
& D_{n} \quad\left\{ \pm\left(e_{i} \pm e_{j}\right): 1 \leq i \neq j \leq n\right\} \\
& \alpha_{j}=e_{j}-e_{j+1} \text { for } j \neq n \\
& \alpha_{n}=e_{n-1}+e_{n}
\end{aligned}
$$

The positive roots associated with the base of simple roots $\Delta$ will be denoted by $\Phi^{+}$, the fundamental dominant weights relative to $\Delta$ will be denoted by $\lambda_{1}, \ldots, \lambda_{n}$, and $\Lambda^{+}$will be the set of all dominant weights. The set $\Lambda^{+}$is in a 1-1 correspondence with $\widehat{G} ; \sigma_{\lambda} \in \widehat{G}$ is indexed by its highest weight $\lambda \in \Lambda^{+}$. The degree of $\sigma_{\lambda}$ will be denoted by $d_{\lambda}$. The weights of $\lambda \in \Lambda^{+}$are given by

$$
\Pi(\lambda)=\{\mu \in \Lambda: w(\mu)<\lambda \text { for all } w \in W\}
$$

where $\mu<\lambda$ means $\lambda-\mu$ is a non-negative integral sum of positive roots. We set $\rho=\sum_{j=1}^{n} \lambda_{j}$. According to the Weyl dimension formula ([9]) the degree of $\lambda$ is given by

$$
\prod_{\alpha \in \Phi^{+}}(\rho+\lambda, \alpha) /(\rho, \alpha)
$$

For general facts about root systems we refer the reader to [4].

A measure $\mu$ on $G$ is called central if $\mu$ commutes with all other measures on $G$ under the action of convolution. Central measures are characterized by the fact that their Fourier transforms are scalar multiples of identity matrices:

$$
\widehat{\mu}(\lambda)=a_{\lambda} I_{d_{\lambda}} \quad \text { where } a_{\lambda}=\int_{G} \frac{\operatorname{Tr} \lambda(x)}{d_{\lambda}} d \mu .
$$

We will simply write $\widehat{\mu}(\lambda)$ in place of $a_{\lambda}$. 
An interesting class of singular, central measures are the orbital measures. The orbital measure $\mu_{g}$, supported on the conjugacy class $C(g)$ containing $g \in G$, is defined by

$$
\int_{G} f d \mu_{g}=\int_{G} f\left(t g t^{-1}\right) d m_{G}(t) \quad \text { for } f \in C(G) .
$$

Orbital measures are continuous if and only if $g \notin Z(G)$. One can easily see that $\widehat{\mu_{g}}(\lambda)=\operatorname{Tr} \lambda(g) / d_{\lambda}$, hence the behaviour of the Fourier transform of orbital measures is determined by the pointwise behaviour of characters.

Since characters are class functions it suffices to know their values on the torus: For $g$ in the torus the Weyl character formula [9] states

$$
\operatorname{Tr} \lambda(g)=\frac{e^{i \rho(g)} \sum_{w \in W} \operatorname{det} w \exp i(\rho+\lambda, w(g))}{\prod_{\alpha \in \Phi^{+}}\left(e^{i \alpha(g)}-1\right)}
$$

where this is understood to be a limit if it is an indefinite form. It is important to identify the zeroes of the denominator and so given $g \in T$ we let

$$
\Phi(g)=\{\alpha \in \Phi: \alpha(g) \in 2 \pi \mathbf{Z}\} \quad \text { and let } \quad \Phi^{+}(g)=\Phi(g) \bigcap \Phi^{+} .
$$

It is easily seen that $\Phi(g)$ is a subroot system of $\Phi$ and that $\Phi^{+}(g)$ is a complete set of positive roots of this subroot system. It is known that $\Phi(g)=\Phi$ if and only if $g \in Z(G)$ (see [1, p. 189]). When $\Phi(g)$ is empty $g$ is called a regular element of $G$.

It was shown in [2] that one can evaluate the Weyl character formula (by considering suitable directional derivatives if $\Phi^{+}(g)$ is not empty) to obtain

$$
\frac{|\operatorname{Tr} \lambda(g)|}{d_{\lambda}}=c(g) \frac{\left|\sum_{w \in W} \operatorname{det} w \prod_{\alpha \in \Phi^{+}(g)}(\rho+\lambda, w(\alpha)) \exp i(\rho+\lambda, w(g))\right|}{\prod_{\alpha \in \Phi^{+}}(\rho+\lambda, \alpha)}
$$

From this one can immediately derive the key formula which we use to make estimates:

$$
\frac{|\operatorname{Tr} \lambda(g)|}{d_{\lambda}} \leq c(g) \frac{\sum_{w \in W}\left|\prod_{\alpha \in \Phi^{+}(g)}(\rho+\lambda, w(\alpha))\right|}{\prod_{\alpha \in \Phi^{+}}(\rho+\lambda, \alpha)} .
$$

In order to use this formula at $g \notin Z(G)$ it is helpful to understand the structures of the proper subroot systems $\Phi(g)$ and their Weyl conjugates. 
Clearly it suffices to analyze those subroot systems which are maximal in the sense that there is no other proper subroot system containing it; we provide a listing of these below for the convenience of the reader. For an explanation on how they are determined see [3].

$$
\begin{array}{ll}
\text { Type } & \text { Maximal subroot systems } \\
A_{n} & A_{n-1}, A_{k} \times A_{n-k}: k, n-k \geq 2 \\
B_{n} & B_{n-1}, D_{n}, A_{1} \times D_{n-1}, D_{k} \times B_{n-k}: k, n-k \geq 2 \\
C_{n} & A_{n-1}, C_{k} \times C_{n-k}: k, n-k \geq 1 \\
D_{n} & D_{n-1}, A_{n-1}, D_{k} \times D_{n-k}: k, n-k \geq 2 .
\end{array}
$$

Here $D_{2}$ is understood to mean $\left\{e_{i} \pm e_{j}\right\}, B_{1}=\left\{e_{i}\right\}, C_{1}=\left\{2 e_{i}\right\} . C_{2}$ and $D_{3}$ are the obvious root systems.

\section{Pointwise values of characters}

The following theorem, which compares the value of a character to its degree, was obtained in [3].

Theorem 3.1 Let $G$ be a compact, connected, simple Lie group of type $A_{n-1}, B_{n}, C_{n}$ or $D_{n}$. For every $g \notin Z(G)$ there is a constant $c(g)$ such that

$$
\left|\frac{\operatorname{Tr} \lambda(g)}{\operatorname{deg} \lambda}\right| \leq c(g)(\operatorname{deg} \lambda)^{-s}
$$

for all $\lambda \in \widehat{G}$ if and only if

$$
s \leq s_{0} \equiv \begin{cases}1 /(n-1) & \text { if } G \text { is type } A_{n-1} \text { or } D_{n} \\ 1 /(2 n-1) & \text { if } G \text { is type } B_{n} \\ 2 /(2 n-1) & \text { if } G \text { is type } C_{n}, n \neq 3 \\ 1 / 3 & \text { if } G \text { is type } C_{3} .\end{cases}
$$

Moreover, there is some $g \notin Z(G)$ such that

$$
\left|\frac{\operatorname{Tr} \lambda(g)}{d_{\lambda}}\right| \geq c(g) d_{\lambda}^{-s_{0}}
$$

for the infinite family of representations $\lambda=m \lambda_{1}, m \in 2 \mathbb{Z}^{+}\left(m \lambda_{3}\right.$ in type $C_{3}$ ).

From the final statement of this theorem it is easy to prove the necessity of $k_{0}$ in the main theorem.

Corollary 3.2 Let $G$ be a compact, connected, simple Lie group of type $A_{n}, B_{n}, C_{n}$ or $D_{n}$. Then there is some $g \notin Z(G)$ such that $\widehat{\mu}_{g}^{k_{0}} \notin l^{2}$. 
Proof. By definition,

$$
\left\|\widehat{\mu}_{g}^{k}\right\|_{2}^{2}=\sum_{\lambda \in \widehat{G}} d_{\lambda}^{2}\left|\frac{\operatorname{Tr} \lambda(g)}{d_{\lambda}}\right|^{2 k},
$$

thus for the choices of $g$ for which Theorem 3.1 is optimal we obtain

$$
\left\|{\widehat{\mu_{g}}}^{k}\right\|_{2}^{2} \geq \sum_{m \text { even }} d_{m \lambda_{i}}^{2}\left|\frac{\operatorname{Tr} m \lambda_{i}(g)}{d_{m \lambda_{i}}}\right|^{2 k} \geq c(g) \sum_{m \text { even }} d_{m \lambda_{i}}^{2-2 k s_{0}}
$$

for $i=3$ in type $C_{3}$ and $i=1$ otherwise. The degrees of the representations $m \lambda_{1}$ are easily seen to be $O\left(m^{n}\right)$ in type $A_{n}, O\left(m^{2 n-1}\right)$ in type $B_{n}$ and $C_{n}$, and $O\left(m^{2 n-2}\right)$ in type $D_{n}$; the degree of $m \lambda_{3}$ in $C_{3}$ is $O\left(m^{6}\right)$. It is a routine exercise to check that

$$
d_{m \lambda_{i}}^{2-2 k_{0} s_{0}}=m^{-1}
$$

and thus ${\widehat{\mu_{g}}}^{k_{0}} \notin l^{2}$.

Now we turn to the problem of proving the sufficiency of this choice of $k_{0}$. Our first step is to show that the pointwise bounds of Theorem 3.1 can be sharpened for many points of $G$.

Proposition 3.3 Suppose $G$ is a classical, compact, connected, simple Lie group. Then

$$
\left|\frac{\operatorname{Tr} \lambda(g)}{\operatorname{deg} \lambda}\right| \leq c(g)(\operatorname{deg} \lambda)^{-s}
$$

for all $\lambda \in \widehat{G}$ provided $G, \Phi^{+}(g)$ and $s$ are as described below:

$$
\begin{array}{llc}
\text { Type } & \text { Type of Subroot System } \Phi^{+}(g) & s \\
A_{n} & A_{k} \times A_{n-k-1} & \frac{1}{n-1} \\
B_{n} & B_{n-1} & \frac{1}{2 n-2} \\
& A_{1} \times D_{n-1} & \frac{1}{n} \\
& D_{k} \times B_{n-k} ; k, n-k \geq 2 & \frac{1}{2 n-4} \\
C_{n} & A_{n-1} & \frac{2}{n+1} \\
& C_{k} \times C_{n-k} ; k, n-k \geq 2 & \frac{2}{2 n-3}\left(\frac{1}{3} ; n=4\right) \\
D_{n} & A_{n-1} ; n \geq 5 & \frac{1}{n-2}\left(\frac{2}{7} ; n=5\right) \\
& D_{k} \times D_{n-k} ; k, n-k \geq 2 & \frac{1}{n-2} .
\end{array}
$$


Proof. The arguments used to show this are similar to those used in [3], but more delicate. We first note that inequality (2.3), together with the Weyl dimension formula (2.1), show that it is sufficient to prove that there is some constant $c=c(g)$ such that for all representations $\lambda$,

$$
\left|\frac{\prod_{\alpha \in \Phi^{\prime+}}(\rho+\lambda, \alpha)}{\prod_{\alpha \in \Phi^{+}}(\rho+\lambda, \alpha)^{1-s}}\right|=\left|\prod_{\alpha \in \Phi^{\prime+}}(\rho+\lambda, \alpha)^{s} \prod_{\alpha \in \Phi^{+} \backslash \Phi^{\prime+}}(\rho+\lambda, \alpha)^{s-1}\right| \leq c
$$

whenever $\Phi^{\prime+}$ is the set of positive roots of a maximal subroot system Weyl conjugate to $\Phi^{+}(g)$. One should observe that the action of the Weyl group preserves the type and basic structure of the subroot system.

Throughout the proof we will assume $\rho+\lambda$ can be expressed in terms of the fundamental dominant weights as $\sum_{i=1}^{n} m_{i} \lambda_{i}$. We will also assume $m_{M}=\max _{i=1, \ldots, n} m_{i}$. The letter $c$ will denote a constant which may vary from one line to another.

The proof proceeds by considering each Lie type separately. We give the details here for type $C_{n}$ and maximal proper subroot system $C_{k} \times C_{n-k}$, $k, n-k \geq 2$ to illustrate the ideas. This subroot system has positive roots

$$
\Phi^{\prime+}=\left\{2 e_{l}, e_{i} \pm e_{j}: i<j, l \in J_{1}\right\} \bigcup\left\{2 e_{l}, e_{i} \pm e_{j}: i<j, l \in J_{2}\right\}
$$

where $J_{1}, J_{2}$ are disjoint subsets of $\{1, \ldots, n\},\left|J_{1}\right|=k,\left|J_{2}\right|=n-k$. By symmetry we may assume $1 \in J_{1}$. Let $\Psi^{+}$be the set of roots in $\Phi^{+}$on the letters $\{2, \ldots, n\}$ and $\Psi^{\prime+}=\Psi^{+} \bigcap \Phi^{\prime+}$. Then (3.1) can be written as $P Q$ where

$$
P \equiv\left(\rho+\lambda, 2 e_{1}\right)^{s} \prod_{j \in J_{1} \backslash\{1\}}\left(\rho+\lambda, e_{1} \pm e_{j}\right)^{s} \prod_{j \in J_{2}}\left(\rho+\lambda, e_{1} \pm e_{j}\right)^{s-1}
$$

and

$$
Q \equiv \prod_{\alpha \in \Psi^{\prime+}}(\rho+\lambda, \alpha)^{s} \prod_{\alpha \in \Psi^{+} \backslash \Psi^{\prime+}}(\rho+\lambda, \alpha)^{s-1} .
$$

As $\Psi^{+}$is a root system of type $C_{n-1}$ containing $\Psi^{\prime+}$ a subroot system of type $C_{k-1} \times C_{n-k}$ (with $k \geq 2, n-k \geq 2$ ) it follows from Theorem 3.1 that $Q$ is bounded if $s \leq 2 /(2 n-3)$ and $n-1 \geq 4$, or if $s \leq 1 / 3$ and $n-1=3$.

Since $\left(\rho+\lambda, e_{1}+e_{j}\right)=O\left(m_{M}\right)$ for all $j$,

$$
P \leq c m_{M}^{s\left(1+2\left(\left|J_{1}\right|-1\right)\right)+\left|J_{2}\right|(s-1)},
$$

and because $2 \leq\left|J_{1}\right|,\left|J_{2}\right| \leq n-2$, this expression is bounded for $s \leq 2 /(2 n-3)$. Modifications of these arguments are needed for types $A_{n}, B_{n}$ and $D_{n}$. 
We will refer to the maximal subroot systems of the proposition as the better maximal subroot systems and the others as the worst maximal subroot systems. To summarize, the worst maximal subroot systems are: type $A_{n-1}$ in $A_{n} ; D_{n}$ in $B_{n} ; C_{1} \times C_{n-1}$ in $C_{n} ; D_{n-1}$ in $D_{n}$ and $A_{3}$ in $D_{4}$. (See the list in section 2 of maximal subroot systems.)

Notice that any proper subroot system is either one of the worst types or is contained in one of the better types. For example, a proper subroot system of $A_{n}$ which is not type $A_{n-1}$ is either contained in one of the better subroot systems $A_{k} \times A_{n-k-1}$, or is contained in a proper subroot system of type $A_{n-1}$. We can show, in fact, that the second case falls within the first. The two maximal subroot systems of $A_{n-1}$ are $A_{n-2}$, which is contained in the better subroot system $A_{1} \times A_{n-2}$, and $A_{k} \times A_{n-1-k-1}$, which is contained in the better subroot system $A_{k} \times A_{n-k-1}$. The arguments for the other types are similar.

Clearly Proposition 3.3 continues to hold if $\Phi^{+}(g)$ is only assumed to be contained in one of the better maximal subroot systems. These observations imply that as long as $\Phi^{+}(g)$ is not one of the worst maximal subroot systems, then there is a constant $c(g)$ such that for all $\lambda \in \widehat{G}$,

$$
\left|\frac{\operatorname{Tr} \lambda(g)}{d_{\lambda}}\right| \leq c(g)\left(d_{\lambda}\right)^{-s}
$$

provided $s$ is as stated in the proposition.

As a consequence, for many orbital measures a stronger result than the main theorem can be proved.

Corollary 3.4 Suppose $G$ is a classical, compact, connected, simple Lie group, $g \notin Z(G)$ and $\Phi^{+}(g)$ is not one of the worst maximal subroot systems. Then ${\widehat{\mu_{g}}}^{k_{0}} \in l^{2}$.

Proof. The previous corollary implies that

$$
\left\|{\widehat{\mu_{g}}}^{k}\right\|_{2}^{2}=\sum_{\lambda \in \widehat{G}} d_{\lambda}^{2}\left|\frac{\operatorname{Tr} \lambda(g)}{d_{\lambda}}\right|^{2 k} \leq c(g) \sum_{\lambda \in \widehat{G}} d_{\lambda}^{2-2 k s}
$$

(for $s$ as in the chart). It was shown in [2, Cor. 9] that

$$
\sum_{\lambda \in \widehat{G}} d_{\lambda}^{t}<\infty
$$

whenever $t<-\operatorname{rank} G /\left|\Phi^{+}\right|$and it is a straightforward calculation to check that $2-2 k_{0} s<-\operatorname{rank} G /\left|\Phi^{+}\right|$. 


\section{Completion of the Proof of the Main Theorem}

In this section we will complete the proof of the main theorem.

Theorem 4.1 Let $G$ be a compact, connected, simple Lie group of type $A_{n}, B_{n}, C_{n}$ or $D_{n}$. Then ${\widehat{\mu_{g}}}^{k} \in l^{2}$ for all continuous orbital measures on $G$ if

$$
k>k_{0} \equiv \begin{cases}n+1 / 2 & \text { if } G \text { is type } A_{n} \\ 2 n-1 / 2 & \text { if } G \text { is type } B_{n} \\ n-1 / 4 & \text { if } G \text { is type } C_{n}, n \neq 3 \\ 13 / 4 & \text { if } G \text { is type } C_{3} \\ n-3 / 4 & \text { if } G \text { is type } D_{n} .\end{cases}
$$

Proof. It remains only to prove this theorem for orbital measures $\mu_{g}$ with $\Phi^{+}(g)$ one of the worst maximal subroot systems: type $A_{n-1}$ in $A_{n}$, type $D_{n}$ in $B_{n}$, type $C_{1} \times C_{n-1}$ in $C_{n}$, type $D_{n-1}$ in $D_{n}$ and type $A_{3}$ in $D_{4}$. The previous method will not work for these orbital measures as the (optimal) pointwise estimates on the trace function are not adequate. Instead we will find an upper bound on the $l^{2}$ norm of ${\widehat{\mu_{g}}}^{k}$ by appealing to (2.3) and using the fact that

$$
\left\|{\widehat{\mu_{g}}}^{k}\right\|_{2}^{2}=\sum_{\lambda \in \widehat{G}} d_{\lambda}^{2}\left|\frac{\operatorname{Tr} \lambda(g)}{d_{\lambda}}\right|^{2 k} \leq c \sum_{\Phi^{\prime}=w(\Phi(g))} \sum_{\lambda \in \widehat{G}} \prod_{\alpha \in \Phi^{\prime+}}(\rho+\lambda, \alpha)^{2} \prod_{\alpha \in \Phi^{+} \backslash \Phi^{\prime+}}(\rho+\lambda, \alpha)^{2-2 k}
$$

where the outer sum is taken over the finitely many subroot systems, $\Phi^{\prime}$, Weyl conjugate to $\Phi(g)$. Thus it suffices to show that for $k>k_{0}$,

$$
\sum_{\lambda \in \widehat{G}}\left(\prod_{a \in \Phi^{\prime+}}(\rho+\lambda, \alpha)^{2} \prod_{\alpha \in \Phi^{+} \backslash \Phi^{\prime+}}(\rho+\lambda, \alpha)^{2-2 k}\right)<\infty
$$

for each such positive subroot system $\Phi^{\prime+}$. This alternate approach will allow us to take advantage of the fact that while $|\operatorname{Tr} \lambda(g)| / d_{\lambda}=O\left(d_{\lambda}^{-s_{0}}\right)$ for certain $\lambda$, it is much smaller for others.

We will continue to adhere to the convention that $m_{M}=\max _{i} m_{i}$, where $\rho+\lambda=\sum m_{i} \lambda_{i}$ and $c$ will denote a constant which may vary. Our strategy requires us to consider each type separately. Again, we will give the details for type $C_{n}$ and sketch the main ideas for the other types.

Type $C_{1} \times C_{n-1}$ in $C_{n}$ : We will leave $C_{3}$ for the reader and proceed inductively. So assume $n \geq 4, k>n-1 / 4$, and $\Phi^{\prime+}$ is a type $C_{1} \times C_{n-1}$ subroot system in type $C_{n}$. It will have positive roots

$$
\left\{2 e_{n_{0}}\right\} \bigcup\left\{e_{i} \pm e_{j}, 2 e_{l}: 1 \leq i<j \leq n, 1 \leq l \leq n: i, j, l \neq n_{0}\right\}
$$

for some $n_{0} \in\{1, \ldots, n\}$. 
Case $M<n_{0}$ : Let $\Psi^{+}$be the set of all positive roots on the letters $\{2, \ldots, n\}$, i.e.,

$$
\Psi^{+}=\left\{e_{i} \pm e_{j}, 2 e_{l}: 2 \leq i<j \leq n, 2 \leq l \leq n\right\}
$$

and let $\Psi^{\prime+}=\Phi^{\prime+} \cap \Psi^{+}$. With this notation we may factor

$$
\prod_{\alpha \in \Phi^{+} \backslash \Phi^{\prime+}}(\rho+\lambda, \alpha)^{2-2 k} \prod_{\alpha \in \Phi^{\prime+}}(\rho+\lambda, \alpha)^{2}
$$

as $P Q$, where

$$
P \equiv\left(\rho+\lambda, e_{1} \pm e_{n_{0}}\right)^{2-2 k}\left(\rho+\lambda, 2 e_{1}\right)^{2} \prod_{j \neq 1, n_{0}}\left(\rho+\lambda, e_{1} \pm e_{j}\right)^{2}
$$

and

$$
Q \equiv \prod_{\alpha \in \Psi^{+} \backslash \Psi^{\prime+}}(\rho+\lambda, \alpha)^{2-2 k} \prod_{\alpha \in \Psi^{\prime+}}(\rho+\lambda, \alpha)^{2} .
$$

Since $M<n_{0}$, we have $\left(\rho+\lambda, e_{1} \pm e_{n_{0}}\right)=O\left(m_{M}\right)$. This ensures that $P \leq c m_{M}^{4 n-2-4 k} \leq c m_{1}^{4 n-2-4 k}$.

Now $\Psi^{\prime+}$ is a type $C_{1} \times C_{n-2}$ subroot system in a type $C_{n-1}$ root system $\Psi^{+}$(on the letters $\left.\{2, \ldots, n\}\right)$. For $\alpha \in \Psi^{+},(\rho+\lambda, \alpha)=\left(\sum_{i=2}^{n} m_{i} \lambda_{i}, \alpha\right)$, thus the induction assumption may be applied to $Q$ to give the conclusion

$$
\sum_{m_{2}, \ldots, m_{n}} \prod_{\alpha \in \Psi^{+} \backslash \Psi^{\prime+}}(\rho+\lambda, \alpha)^{2-2 k} \prod_{\alpha \in \Psi^{\prime+}}(\rho+\lambda, \alpha)^{2}<\infty .
$$

Since $4 n-2-4 k<-1$, we can combine these facts to obtain

$$
\begin{aligned}
& \sum_{\lambda: M<n_{0}} \prod_{\alpha \in \Phi^{+} \backslash \Phi^{\prime+}}(\rho+\lambda, \alpha)^{2-2 k} \prod_{\alpha \in \Phi^{\prime+}}(\rho+\lambda, \alpha)^{2} \\
\leq & c \sum_{m_{1}} m_{1}^{4 n-2-4 k} \sum_{m_{2}, \ldots, m_{n}} \prod_{\alpha \in \Psi^{+} \backslash \Psi^{\prime+}}(\rho+\lambda, \alpha)^{2-2 k} \prod_{\alpha \in \Psi^{\prime+}}(\rho+\lambda, \alpha)^{2}<\infty .
\end{aligned}
$$

Type $A_{n-1}$ in $A_{n}$ or Type $D_{n-1}$ in $D_{n}$ : Before explaining the case $M \geq n_{0}$ we remark that a similar induction argument works for these two types, as well. We again factor the summands in (4.1) as $P Q$, where bounds on $Q$ can be obtained by the induction assumption and $P$ can be bounded by finding a suitable number of roots $\alpha$ with $(\rho+\lambda, \alpha) \geq m_{M}$. For type $A_{n}$ we take $Q$ to be the product of the factors involving positive roots built on either the letters $\{2, \ldots, n+1\}$ or $\{1, \ldots, n\}$, depending on whether $M<n_{0}$ or $M \geq n_{0}$ (giving the problem of type $A_{n-2}$ in $A_{n-1}$ ). In the first case we can use the fact that $(\rho+\lambda, \alpha) \geq m_{M}$ for $\alpha=e_{1}-e_{n_{0}}$ to bound $P$. In the second case, the root $\alpha=e_{n_{0}}-e_{n+1}$ has the desired property. 
For type $D_{n}$ one can observe that it suffices to assume $n_{0}>1$ and thus we may apply an induction argument by taking $Q$ to be the product of the factors involving the roots built on the letters $\{2, \ldots, n\}$. Finding a suitable number of roots $\alpha$ with $(\rho+\lambda, \alpha) \geq m_{M}$ is somewhat more delicate in this case. For example, if $M \geq n_{0}$, then $(\rho+\lambda, \alpha) \geq m_{M}$ for $\alpha=e_{i}+e_{n_{0}}$ for all $i \neq n_{0}\left(\right.$ or $\left.i \neq n_{0}, n\right)$ provided $M \neq n-1(M=n-1)$ and for $\alpha=e_{n_{0}}-e_{n}$ if $M=n-1$.

Type $C_{1} \times C_{n-1}$ in $C_{n}$ (ctd):

Case $M \geq n_{0}$ : Here we take a different approach and instead factor

$$
\prod_{\alpha \in \Phi^{+} \backslash \Phi^{\prime+}}(\rho+\lambda, \alpha)^{2-2 k} \prod_{\alpha \in \Phi^{\prime+}}(\rho+\lambda, \alpha)^{2}
$$

as $P^{\prime} Q^{\prime}$ where

$$
P^{\prime}=\prod_{i \neq n_{0}}\left(\rho+\lambda, e_{i}+e_{n_{0}}\right)^{2-2 k} \prod_{i}\left(\rho+\lambda, 2 e_{i}\right)^{2} \prod_{i<j ; i, j \neq n_{0}}\left(\rho+\lambda, e_{i}+e_{j}\right)^{2}
$$

and

$$
Q^{\prime}=\prod_{i \neq n_{0}}\left(\rho+\lambda, e_{i}-e_{n_{0}}\right)^{2-2 k} \prod_{i<j ; i, j \neq n_{0}}\left(\rho+\lambda, e_{i}-e_{j}\right)^{2} .
$$

Note that $\left(\rho+\lambda, e_{i}-e_{j}\right)=\left(\sum_{k=1}^{n-1} m_{k} \lambda_{k}, e_{i}-e_{j}\right)$ for any $j>i$. Thus $Q^{\prime}$ is the summand in (4.1) which arises from a subroot system of type $A_{n-2}$ in $A_{n-1}$, on the letters $\{1, \ldots, n\}$. As $k>n-1 / 2$ it follows from the analysis for this case that

$$
\sum_{m_{1}, \ldots, m_{n-1}} \prod_{i \neq n_{0}}\left(\rho+\lambda, e_{i}-e_{n_{0}}\right)^{2-2 k} \prod_{i<j ; i, j \neq n_{0}}\left(\rho+\lambda, e_{i}-e_{j}\right)^{2}<\infty .
$$

Thus it suffices to prove $P^{\prime} \leq \mathrm{cm}_{n}^{-t}$ for some $t>1$, and this is what we will verify. It is convenient to let $b_{i}=\max _{l \geq i} m_{l}$. Then $\left(\rho+\lambda, 2 e_{i}\right)$ and $\left(\rho+\lambda, e_{i}+e_{j}\right)$ for $j>i$ are $O\left(b_{i}\right)$, so

$$
\begin{aligned}
P^{\prime} & \leq c \prod_{j<n_{0}} b_{j}^{2-2 k} \prod_{j>n_{0}} b_{n_{0}}^{2-2 k} \prod_{i} b_{i}^{2} \prod_{i<j ; i, j \neq n_{0}} b_{i}^{2} \\
& =c \prod_{i<n_{0}} b_{i}^{2(n-i-1)+4-2 k} \prod_{i>n_{0}} b_{i}^{2+2(n-i)}\left(b_{n_{0}}^{2+(2-2 k)\left(n-n_{0}\right)}\right) .
\end{aligned}
$$

Now $b_{i} \leq m_{M}$ and $2+2(n-i)>0$, thus

$$
\prod_{i>n_{0}} b_{i}^{2+2(n-i)} \leq \prod_{i>n_{0}} m_{M}^{2+2(n-i)}=m_{M}^{\left(n-n_{0}+1\right)\left(n-n_{0}\right)} .
$$

Since $M \geq n_{0}, b_{i}=m_{M}$ for $i \leq n_{0}$, hence

$$
\prod_{i<n_{0}} b_{i}^{2(n-i-1)+4-2 k}=m_{M}^{\left(2 n+2-2 k-n_{0}\right)\left(n_{0}-1\right)} .
$$


Combining these observations, and using the fact that $n \geq 4$, gives the bound

$$
P^{\prime} \leq c m_{M}^{2 k+n^{2}+n-2 k n} \leq c m_{n}^{-5 / 2},
$$

completing the proof of this case.

Type $D_{n}$ in $B_{n}$ : In this case $\Phi^{+} \backslash \Phi^{\prime+}$ consists of all the positive roots of length one. If $\alpha=e_{i}$ is such a root, then

$$
(\rho+\lambda, \alpha)=O\left(m_{i}+\cdots+m_{n}\right) \geq(\rho+\lambda, \beta)
$$

for $\beta=e_{i} \pm e_{j}$ and any $i<j$. From this it follows that (4.1)

$$
\begin{aligned}
& \sum_{\lambda} \prod_{\alpha \in \Phi^{+} \backslash \Phi^{\prime+}}(\rho+\lambda, \alpha)^{2-2 k} \prod_{\alpha \in \Phi^{\prime+}}(\rho+\lambda, \alpha)^{2} \\
& \leq c \sum_{m_{1}, \ldots, m_{n}}\left(\prod_{i=1}^{n}\left(m_{i}+\cdots+m_{n}\right)\right)^{4 n-4 i-2 k+2} \leq c \sum_{m_{1}, \ldots, m_{n}}\left(\prod_{i=1}^{n} m_{i}\right)^{4 n-2 k-2},
\end{aligned}
$$

and this is finite as $4 n-2 k-2<-1$.

Type $A_{3}$ in $D_{4}$ : This type can be done by explicit calculation. Alternatively, one can effectively reduce the problem to that of type $D_{3}$ in $D_{4}$ by considering a suitable automorphism of the root system.

Remark 4.1 The main theorem stated in the introduction results from Corollary 3.2 and Theorem 4.1. It would be interesting to know if there was a less computational proof of this result, or a proof which did not involve consideration of each Lie type separately. Also, it remains to carry out the analysis for the exceptional groups. The maximal subroot systems of $E_{6}, E_{7}$ and $E_{8}$ seem to be too cumbersome for our approach.

\section{Applications}

\subsection{Convolutions}

Since the convolution of two $L^{2}$ functions is continuous we immediately obtain the following corollary of the theorem.

Corollary 5.1 If $\mu$ is a continuous orbital measure, then $\mu^{2 n-1}$ is a continuous function if $G$ is type $A_{n-1}$ or $D_{n}, \mu^{2 n}$ is continuous if $G$ is type $C_{n}$, $n \geq 4$, and $\mu^{4 n-1}$ is a continuous function if $G$ is type $B_{n}$.

A similar result will hold for any central, continuous measure compactly supported on the conjugates of a set of the form $\left\{x \in T: \Phi^{+}(x)=\Phi^{+}\right\}$for some fixed set $\Phi^{+}$(c.f., [2, Cor. 7]). 


\section{2. $\mathrm{L}^{p}$-Improving Measures}

A measure $\mu$ is called $L^{p}$-improving if there is some $p<2$ such that

$$
\mu * L^{p} \subseteq L^{2} .
$$

Young's inequality implies that all functions in $L^{q}$, for some $q>1$, are examples of $L^{p}$-improving measures. A question of current interest is to understand which singular measures on compact groups are $L^{p}$-improving.

In [6] surface measures on compact, connected analytic submanifolds which generate the group were shown to be $L^{p}$-improving. Continuous orbital measures are an example of this phenomena. The measure $\mu_{g}$ is supported on the conjugacy class containing $g$, and this manifold generates $G$ since a suitable $k$-fold product supports the non-zero, absolutely continuous measure $\mu_{g}^{k}$ and thus must have positive Haar measure.

A more refined problem is to determine the minimal $p$ such that $\mu * L^{p} \subseteq$ $L^{2}$. This was done for for orbital measures corresponding to regular elements (those with $\Phi^{+}(g)$ empty) in [7] where it was shown that

$$
\mu_{g} * L^{p} \subseteq L^{2} \quad \text { if and only if } \quad p \geq 1+r /(2 \operatorname{dim} G-r) .
$$

Our theorem gives results for all continuous orbital measures.

Proposition 5.2 If $g \notin Z(G)$ then $\mu_{g} * L^{p} \subseteq L^{2}$ for $p>p_{0}$ where:

(i) $p_{0}=2-4 /(2 n+3)$ when $G$ is type $A_{n}$;

(ii) $p_{0}=2-4 /(4 n+1)$ when $G$ is type $B_{n}$;

(iii) $p_{0}=2-8 /(4 n+3)$ when $G$ is type $C_{n}, n \neq 3 ; p_{0}=26 / 17$ if $n=3$;

(iv) $p_{0}=2-8 /(4 n+1)$ when $G$ is type $D_{n}$.

Proof. The main theorem implies that for $k>k_{0}$ the operator

$$
T_{k}(f)=\mu_{g}^{k} * f
$$

maps $L^{1}(G)$ into $L^{2}(G)$ whenever $g \notin Z(G)$. Since the identity map obviously maps $L^{2}(G)$ into $L^{2}(G)$, an application of Stein's interpolation theorem [8] gives that $\mu_{g} * L^{p} \subseteq L^{2}$ for the choices of $p_{0}$ listed.

Remark 5.1 It would be interesting to know if these results are optimal for the orbital measures corresponding to the worst subroot systems. Clearly they are not optimal for other continuous, orbital measures as better results can be obtained by invoking Proposition 3.3. 


\section{References}

[1] BröckeR, T. AND Dieck, T.: Representations of compact Lie groups. Graduate Texts in Mathematics 98. Springer-Verlag, New York, 1985.

[2] Hare, K. E.: The size of characters of compact Lie groups. Studia Math. 129 (1998), 1-18.

[3] Hare, K. E., Wilson, D. And Yee, W. L.: Pointwise estimates of the size of characters of compact Lie groups. J. Austral. Math. Soc. Ser. A. 69 (2000), 61-84.

[4] Humphreys, J. E.: Introduction to Lie algebras and representation theory. Graduate Texts in Mathematics 9. Springer-Verlag, New York-Berlin, 1972.

[5] Ragozin, D. L.: Central measures on compact simple Lie groups. J. Functional Analysis 10 (1972), 212-229.

[6] Ricci, F. And Stein, E. M.: Harmonic analysis on nilpotent groups and singular integrals. III. Fractional integration along manifolds. J. Funct. Anal. 86 (1989), 360-389.

[7] Ricci, F. and Travaglini, G.: $L^{p}-L^{q}$ estimates for orbital measures and Radon transform on compact Lie groups and Lie algebras. J. Funct. Anal. 129 (1995), 132-147.

[8] Stein, E. M. And Weiss, G.: Introduction to Fourier analysis on Euclidean spaces. Princeton Mathematical Series 32. Princeton University Press, Princeton, N.J., 1971.

[9] Varadarajan, V.S.: Lie groups, Lie algebras, and their representations. Reprint of the 1974 edition. Graduate Texts in Mathematics 102. SpringerVerlag, New York, 1984.

Recibido: 27 de septiembre de 2002

Kathryn E. Hare Department of Pure Mathematics

University of Waterloo 200 University Avenue W.

N2L 3G1, Waterloo, Ontario (Canada) kehare@math. uwaterloo.ca

Wai Ling Yee Department of Mathematics MIT 02139, Cambridge, MA (USA) wlyee@math.mit.edu

This research was supported in part by NSERC. 